Marc Debus

\title{
Regierungsbildung, Ämterverteilung und Politikinhalte nach der Bundestagswahl 2009: Wer hat sich in der schwarz-gelben Koalition am besten durchgesetzt?
}

\section{Einleitung und Fragestellung}

Die Bundestagswahl 2009 hat - entgegen manchen Erwartungen - zu keiner Widerauflage der Koalition aus Christdemokraten und Christsozialen (CDU/CSU) auf der einen und Sozialdemokraten (SPD) auf der anderen Seite geführt. Vielmehr konnte ein Bündnis aus CDU/CSU und Freidemokraten (FDP) eine deutliche Mehrheit der Bundestagsmandate erreichen, so dass sich im Gegensatz zu 2005 eine Regierung aus solchen Parteien bilden konnte, die bereits im Wahlkampf aufgrund einer wechselseitigen Koalitionsaussage die Bereitschaft zum gemeinsamen Regieren bekräftigt hatten. ${ }^{1}$ Da dementsprechend die Koalitionsbildung wenig spannend verlief, gewann die Verteilung der Kabinettsposten auf die drei Koalitionsparteien CDU, CSU und FDP sowie die inhaltlichen Kompromisse, auf die sich Union und Liberale geeinigt haben, deutlich größere Beachtung. Die Freien Demokraten konnten mit fünf von 15 Ministern einen - gemessen an der Sitzzahl, die sie zur Mehrheit der Koalition beitragen - leicht überdurchschnittlichen Anteil an Kabinettsposten gewinnen.

Gilt dies auch für die Verteilung der parlamentarischen Staatssekretäre? Konnte sich die FDP auch inhaltlich überdurchschnittlich stark in den Koalitionsverhandlungen durchsetzen? Sind die Liberalen in der gegenwärtigen Konstellation des bundesdeutschen Parteienwettbewerbs überhaupt die Partei, die über ein überdurchschnittliches Verhandlungspotential verfügt? In diesem Beitrag soll zunächst der Frage nachgegangen werden, welche der beiden Regierungsparteien theoretisch über einen Vorteil in den Koalitionsverhandlungen 2009 verfügt hat. Dazu wird auf das von Norman Schofield entwickelte Modell des »Political Heart $\ll^{2}$ zurückgegriffen, das bereits öfters zur Analyse von Parteienwettbewerb und Koalitionsbildung in Deutschland verwendet worden

1 Vgl. Frank Decker, »Koalitionsaussagen der Parteien vor Wahlen. Eine Forschungsskizze im Kontext des deutschen Regierungssystems « in: Zeitschrift für Parlamentsfragen 40 (2009), H. 2, S. 444-448.

2 Norman Schofield, »Political competition and multiparty coalition governments « in: European Journal of Political Research 23 (1993), H. 1, S. 1-33; Norman Schofield, »Coalition Politics: A Formal Model and Empirical Analysis « in: Journal of Theoretical Politics 7 (1995), H. 3, 245-281. 
ist. ${ }^{3}$ Im Gegensatz zu seiner »reinen « Form, die lediglich Informationen zur Stärke der Parteien im Parlament und deren programmatischen Positionen in einem zweidimensionalen Politikraum umfasst, wird das Modell des »Political Heart« im vorliegenden Beitrag in der Form erweitert, dass kontextuelle Informationen des Parteienwettbewerbs im Sinne von Koalitionsaussagen mit einbezogen werden. Zahlreiche jüngere Studien weisen auf die Bedeutung von Vorwahlkoalitionen für Wahlverhalten, Parteienwettbewerb und die Muster der Regierungsbildung in Deutschland wie auch im internationalen Vergleich hin. ${ }^{4}$ Auf der Grundlage dieses modifizierten »Political Hearts « ist es möglich, nicht nur die Parteien zu bestimmen, die die künftige Regierung bilden sollten, sondern auch die Partei zu identifizieren, die sich in den Koalitionsverhandlungen am besten durchsetzen sollte. Aufbauend darauf soll im zweiten Schritt anhand einer Analyse sowohl der Ämterverteilung als auch der von Union und FDP in Form des Koalitionsabkommens formulierten künftigen Politikziele analysiert werden, ob der vorhergesagte zentrale parteipolitische Akteur des Koalitionsspiels nach den Bundestagswahlen 2009 sich tatsächlich auch in Fragen der Ämterverteilung wie auch der Politikinhalte am besten durchgesetzt hat. Die Ergebnisse zeigen, dass aufgrund der Fraktionsstärken, der politikfeldspezifischen Positionen sowie der vor den Wahlen getätigten Koalitionsaussagen der Parteien CDU und CSU sich am besten in den Koalitionsverhandlungen hätten durchsetzen sollen. Dies gelang der Union jedoch nur teilweise: so etwa bei der Gewinnung eines überdurchschnittlichen Anteils von parlamentarischen Staatssekretären sowie der Entsendung von parlamentarischen Staatssekretären als »watch-dogs ${ }^{5}$ in zwei von FDP-Mitgliedern kontrollierten Ministerien. Die ermittelte Position des Koalitionsabkommens macht hingegen deutlich, dass die Liberalen sich nicht überdurchschnittlich in ihrem zentralen Politikfeld der Wirtschafts-, Sozial- und Finanzpolitik, sondern vor allem in der Innen-, Rechts- und Gesellschaftspolitik durchsetzen konnten, so dass in sozioökonomischen Fragen eher eine moderate Reformpolitik, die nahe an der wirtschafts-

3 Vgl. etwa Franz U. Pappi, »Regierungsbildung im deutschen Fünf-Parteiensystem« in: Politische Vierteljabresschrift 50 (2009), H. 2, S. 187-202; Eric Linhart, Ampel, Linkskoalition und Jamaika als Alternativen zur großen Koalition. MZES-Arbeitspapier Nr. 91. Mannheim.

4 So etwa Lanny W. Martin / Randolph T. Stevenson, »Government Formation in Parliamentary Democracies « in: American Journal of Political Science 45 (2001), H. 1, S. 33-50; Franz U. Pappi / Thomas Gschwend, »Partei- und Koalitionspräferenzen der Wähler bei den Bundestagswahlen 1998 und 2002« in: Jürgen W. Falter / Oscar W. Gabriel / Bernhard Wessels (Hrsg.), Wablen und Wähler. Analysen aus Anlass der Bundestagswabl 2002. Wiesbaden 2005, S. 284-305; Eric Linhart, »Rationales Wählen als Reaktion auf Koalitionssignale am Beispiel der Bundestagswahl 2005 « in: Politische Vierteljabresschrift 48 (2007), H. 3, S. 461-484; Thomas Bräuninger / Marc Debus, »Der Einfluss von Koalitionsaussagen, programmatischen Standpunkten und der Bundespolitik auf die Regierungsbildung in den deutschen Ländern « in: Politische Vierteljabresschrift 49 (2008), H. 2, S. 309-338; Marc Debus, »Pre-electoral commitments and government formation« in: Public Choice 138 (2009), H. 1-2, S. 45-64; Frank Decker, a.a.O. (Fußnote 1), S. 435-436.

5 Michael F. Thies, »Keeping Tabs on Partners: The Logic of Delegation in Coalition Governments « in: American Journal of Political Science, 45. Jg (2001), H. 3, S. 580-598; vgl. Luca Verzichelli, »Portfolio Allocation « in: Kaare Strøm / Wolfgang C. Müller / Torbjörn Bergman (Hrsg.), Cabinet Governance: Bargaining and The Democratic Life Cycle in Western Europe. Oxford 2008, S. 237-267. 
politischen Position der Christdemokraten liegt, für die 17. Legislaturperiode des Deutschen Bundestages zu erwarten ist.

Um zu diesen Schlussfolgerungen zu gelangen wird zunächst ein Überblick zum Stand der Forschung hinsichtlich der Bestimmung von zentralen Akteuren im Koalitionsspiel sowie den Mustern von Ämterverteilung und inhaltlichen Kompromissen zwischen den Regierungsparteien gegeben. Im Anschluss daran wird näher auf das hier herangezogene Modell zur Bestimmung der durchsetzungsstärksten Partei und die hier vorgenommenen Modifikationen des »Political Heart«-Modells eingegangen. Bevor die Ergebnisse des Modelltests präsentiert werden, wird jedoch zunächst dargelegt, mit welcher Methode die Policy-Positionen der Bundestagsparteien einerseits und diejenigen des Koalitionsabkommens andererseits bestimmt werden. Die Schlussbetrachtung fasst die gewonnenen Erkenntnisse zusammen und diskutiert Anreize für weitergehende Forschungsfragen.

\section{Literaturüberblick}

\subsection{Schlüsselakteure in Koalitionsbildungsprozessen}

Die umfangreiche Literatur zur Koalitionsbildung bietet zahlreiche Ansätze, die es ermöglichen, den entscheidenden Akteur in der Koalitionsbildungsphase einerseits sowie im Zeitraum der Koalitionsverhandlungen andererseits zu bestimmen. Generell lassen sich Theorien der Koalitionsbildung in eine an der Besetzung möglichst vieler politischer Ämter ausgerichtete Perspektive einerseits und einen sich auf politische Inhalte konzentrierenden Blickwinkel andererseits unterteilen. ${ }^{6}$ Aus der reinen office-orientierten Perspektive sagt etwa die Theorie des »dominanten Spielers « von Peleg ${ }^{7}$ voraus, dass die Partei, die häufiger als andere Parlamentsfraktionen an allen potentiellen Mehrheitskoalitionen beteiligt ist, Teil der künftigen (Koalitions-)Regierung sein wird. Dieser »dominant player « ist Peleg zu Folge immer die stärkste Partei im Parlament. ${ }^{8}$

Berücksichtigt man zusätzlich dazu den an Politikinhalten ausgerichteten Ansatz, so sollten die ideologischen Ausrichtungen bzw. die politikfeldspezifischen Positionen der Parteien von Bedeutung für die Muster der Koalitionsbildung und damit auch für die Identifikation von Schlüsselakteuren - so genannten »key players « oder »pivotal par-

6 Für einen Überblick vgl. Michael Laver / Norman Schofield, Multiparty Government. The Politics of Coalition in Europe. Ann Arbor 1998; Wolfgang C. Müller, »Government formation « in: Todd Landmann / Neil Robinson (Hrsg.), The SAGE Handbook of Comparative Politics. Los Angeles 2009, S. 227-245.

7 Bazalel Peleg, »Coalition Formation in Simple Games with Dominant Players « in: International Journal of Game Theory 10 (1981), H. 1, S. 11-33; vgl. Adrianus van Deemen, »Dominant players and minimum size coalitions « in: European Journal of Political Research 17 (1989), H. 3, S. 313-332.

8 Vgl. Bazalel Peleg, a.a.O. (FN 7), S. 12-13. 
ties « ${ }^{9}-$ im Koalitionsspiel sein. Aufbauend auf dem »median voter «-Theorem von Downs ${ }^{10}$ sowie den Arbeiten von Peleg und van Deemen ${ }^{11}$ kann van Roozendaal ${ }^{12}$ theoretisch herleiten und am Beispiel der niederländischen Regierungsbildungen von 1918 bis 1990 zeigen, dass die Partei, die über den Median-Abgeordneten im Parlament verfügt, eine zentrale Stellung im Koalitionsspiel einnimmt und sicher Teil der künftigen Regierungskoalition sein wird. Dazu ordnet van Roozendaal die niederländischen Parteien auf einer allgemeinen Links-Rechts-Dimension an und bestimmt anhand der Sitzstärke der Fraktionen, welche Partei den Abgeordneten umfasst, der zwischen Mehrheit und Minderheit im Parlament auf dieser Achse unterscheidet. ${ }^{13}$

Nun beschreiben eindimensionale Politikräume in den meisten Fällen nur unzureichend die Muster des Parteienwettbewerbs in vielen modernen Demokratien. Aus politisch-soziologischen Studien wissen wir, dass in der Regel mehr als eine soziale Konfliktlinie (»cleavage«) die Muster des Parteienwettbewerbs beeinflusst. ${ }^{14}$ Neben dem sozioökonomisch determinierten Gegensatz zwischen Arbeitnehmern und Kapitaleignern ist dies in vielen europäischen Ländern der Konflikt zwischen säkular-liberalen Auffassungen einerseits und klerikal-konservativen Positionen in gesellschaftspolitischen Fragen andererseits. ${ }^{15}$ Daher sind Modelle zur Bestimmung von pivotalen parteipolitischen Akteuren in Regierungsbildungsprozessen notwendig, die zumindest zwei Politikdimensionen berücksichtigen. Einen solchen Ansatz stellt das »Political Heart«-Modell von Schofield dar. ${ }^{16}$ Analog zum Modell ministerieller Diskretion von Laver und Sheps$1 \mathrm{e}^{17}$ werden die Positionen der im Parlament vertretenen Parteien in einem zweidimensionalen Politikraum verortet, wobei - im Gegensatz zum theoretischen Ansatz von Laver und Shepsle - jedoch die jeweils zwischen Mehrheit und Minderheit trennenden Me-

9 Vgl. Hans Keman, »Koalitionen in Belgien und in den Niederlanden: Spiegel des Wandels von Konkordanz- zu moderaten Konsensdemokratien « in: Sabine Kropp, Suzanne S. Schüttemeyer / Roland Sturm (Hrsg.), Koalitionen in West- und Osteuropa. Opladen 2002, S. 107-135.

10 Anthony Downs, An Economic Theory of Democracy. New York 1957.

11 Vgl. Bazalel Peleg, a.a.O. (FN 7); Adrianus van Deemen, a.a.O. (FN 7).

12 Peter van Roozendaal, Cabinets in the Netherlands (1918-1990): »The Importance of >Dominant< and >Centrak Parties « in: European Journal of Political Research 23 (1993), H. 1, S. 3554; vgl. Adrianus van Deemen, Coalition Formation in Centralized Policy-Games « in:Journal of Theoretical Politics 3 (1991), H. 2, S. 139-161.

13 Vgl. Peter van Roozendaal, a.a.O. (FN 12).

14 Seymour Martin Lipset / Stein Rokkan, Cleavage Structures, »Party Systems and Voter Alignments: An Introduction« in: Seymour M. Lipset / Stein Rokkan (Hrsg.), Party Systems and Voter Alignments: Cross-National Perspectives. New York, London 1967, S. 1-64; vgl. Michael Laver / W. Ben Hunt, Policy and Party Competition. London, New York 1992, S. 58; Franz U. Pappi, a.a.O. (FN 3), S. 195.

15 Vgl. Michael Laver / W. Ben Hunt, a.a.O. (FN 14); Paul V. Warwick, »Toward a Common Dimensionality in West European Policy Spaces « in: Party Politics 8 (2002), H. 1, S. 101-122; Paul V. Warwick, Policy horizons and parliamentary government. Houndmills 2006; Kenneth Benoit / Michael Laver, Party Policy in Modern Democracies. London, New York 2006.

16 Vgl. Norman Schofield, a.a.O. (FN 2).

17 Michael Laver / Kenneth A. Shepsle, »Coalitions and Cabinet Government « in: American Political Science Review 84 (1990), H. 4, S. 873-890; Michael Laver / Kenneth A. Shepsle, Making and Breaking Governments. Cambridge 1996. 
dianlinien ausschlaggebend sind. Schneiden sich die Medianlinien in einem Punkt, so verfügt die entsprechende Parteienkonstellation über einen »Kern«. Die Partei an der Stelle dieses »core« sollte demnach auf jeden Fall die Regierung bilden und - ceteris paribus - sich aufgrund dieser Schlüsselrolle in den Koalitionsverhandlungen am besten durchsetzen können. Sollten sich die Medianlinien nicht in einem Punkt schneiden, dann ergibt sich ein »Political Heart« bzw. die so genannte Zyklusmenge. ${ }^{18}$ Das »Political Heart « ist die vollständig von Medianlinien begrenzte Fläche. Die Parteien, die das »Heart « bilden, nehmen eine zentrale Stellung im Regierungsbildungsprozess ein. Jedoch können die Voraussagen für Parteiensysteme mit einer geringen Anzahl programmatischer Akteure bei Heranziehung des »Political Heart«-Modells rasch trivial werden: sollte sich in einem hypothetischen Drei-Parteiensystem kein Core ergeben, dann wären alle drei Parteien Teil der Zyklusmenge und damit zentrale Akteure im Koalitionsbildungsprozess. Der Informationsgewinn, den Schofields Modell in diesem Fall liefern würde, wäre gleich Null. Bevor im theoretischen Abschnitt dieses Aufsatzes ein Lösungsvorschlag für das letztgenannte Problem präsentiert wird, soll zunächst auf den Stand der Forschung zu den Mustern und Determinanten von Ämterverteilung und Politikzielen in Koalitionsregierungen eingegangen werden.

\section{2 Ämterverteilung und Politikkompromisse in Koalitionsregierungen}

Zur Beantwortung der Frage, welche Partei sich innerhalb einer Koalitionsregierung am besten hat durchsetzen können, wird von politischen Beobachtern wie auch in der Politikwissenschaft in der Regel die Ämterverteilung im Kabinett herangezogen. Hierbei gilt die von Gamson ${ }^{19}$ zuerst festgestellte und in zahlreichen späteren Studien immer wieder nachgewiesene Proportionalitätsnorm: eine Regierungspartei erhält einen ungefähr so hohen Anteil an Ministerien, wie sie zur Mehrheit der Koalition im Parlament beiträgt. Obwohl dieser Zusammenhang nicht perfekt ist, so erreicht er doch für politikwissenschaftliche Verhältnisse sehr hohe Korrelationswerte, wodurch die Beobachtung von Gamson häufig in den Status eines »Gesetzes « befördert wird. ${ }^{20}$ Browne und Franklin konnten in einer frühen Replikationsstudie zeigen, dass kleinere Regierungsparteien der Tendenz nach einen überdurchschnittlich hohen Anteil an Kabinettsposten erreichen. ${ }^{21}$ Generell gilt aber, dass selbst unter Kontrolle der Bedeutung der jeweiligen Ministerien eines Landes die Proportionalitätsnorm weitgehend erhalten bleibt. ${ }^{22}$

18 Vgl. für eine Anwendung auf den deutschen Parteienwettbewerb Franz U. Pappi, a.a.O. (FN 3).

19 William Gamson, »A Theory of Coalition Formation« in: American Sociological Review 26 (1961), H. 3, S. 373-382.

20 Vgl. Michael Laver / Norman Schofield, a.a.O. (Fußnote 6), S. 171-181.

21 Eric C. Browne / Mark N. Franklin, »Aspects of Coalition Payoffs in European Parliamentary Democracies in: American Political Science Review 67 (1973), H. 2, S. 453-469.

22 Paul V. Warwick / James N. Druckman, »Portfolio Salience and the Proportionality of Payoffs in Coalition Governments « in: British Journal of Political Science 31 (2006), H. 4, S. 627-649; Royce Carroll / Gary W. Cox, »The logic of Gamson's law: Pre-electoral coalitions and portfolio allocations « in: American Journal of Political Science 51 (2007), H. 2, S. 300-313; Hanna 
Browne und Feste ${ }^{23}$ sowie Budge und Keman ${ }^{24}$ haben die Ämterverteilung in Koalitionsregierungen weniger unter dem numerischen Aspekt als vielmehr aus inhaltlicher Perspektive betrachtet. Unter Heranziehung des Parteifamilienansatzes ${ }^{25}$ haben Budge und Keman eine Reihenfolge der Ministerien gebildet, die zwischen solchen Ämtern unterscheidet, die für Parteien aus fünf Parteifamilien - der konservativen, der liberalen, der christlich-religiösen, der sozialistischen und der agrarischen Parteifamilie - zwischen wichtig und unwichtig verläuft. ${ }^{26}$ Parteien mit unterschiedlichem historisch-ideologischem Hintergrund versuchen solche Ministerien zu besetzen, von der sie sich den größtmöglichen Nutzen zur Befriedigung der Präferenzen ihrer Anhänger versprechen. So stellen etwa in rund 80 \% aller Kabinette, an denen sozialistische oder sozialdemokratische Parteien beteiligt sind, parteipolitische Gruppen aus dieser Parteifamilie das Ministerium, das für Arbeit, Gesundheit und Soziales zuständig ist. Unter Bezugnahme auf den Datensatz des Comparative Manifesto Project (CMP) ${ }^{27}$ kann zudem gezeigt werden, dass selbst unter Kontrolle der Sitzstärke und des Parteifamilienansatzes die in den Wahlprogrammen seitens der Parteien geäußerten Issue-Salienzen einen Effekt auf die Ämterverteilung in Koalitionsregierungen ausüben: je wichtiger einer Koalitionspartei ein mit einem Ministerium verwandtes Thema ist, desto wahrscheinlicher wird sie es im Zuge der Verhandlungen über die Regierungsbildung auch erhalten. ${ }^{28}$

Zahlreiche an den Mustern der Ämterverteilung ausgerichtete Studien beziehen neben der Anzahl der Ministerien auch die Anzahl der so genannten »junior ministers « mit ein. ${ }^{29}$ Hintergrund der Überlegung ist, dass diese von einer Koalitionspartei in solchen Ministerien platziert werden, in denen der Minister vom jeweiligen Regierungspartner kommt. Thies findet empirische Evidenz für das Argument, dass Juniorminister - in Deutschland die parlamentarischen Staatssekretäre - als »watch dogs « von den Regie-

Bäck / Thomas Persson / Henk Erik Meier, »Party Size and Portfolio Payoffs: A Study of the Mechanism Underlying the Proportionality Relationship in Coalition Governments « in: Journal of Legislative Studies 15 (2009), H. 1, S. 10-34.

23 Eric C. Browne / Karen Feste, "Qualitative Dimensions of Coalition Payoffs « in: American Behavioral Scientist 18 (1975), H. 4, S. 530-556.

24 Ian Budge / Hans Keman, Parties and Democracy. Coalition Formation and Government Functioning in Twenty States. Oxford 1990.

25 Peter Mair / Cas Mudde, »The Party Family and its Study « in: Annual Review of Political Science 1 (1998), S. 211-229; Michael Gallagher / Michael Laver / Peter Mair, Representative government in modern Europe. New York 2006, S. 230-262.

26 Ian Budge / Hans Keman, a.a.O. (FN 24), S. 90.

27 Ian Budge / Hans-Dieter Klingemann / Andrea Volkens / Judith Bara / Eric Tanenbaum (Hrsg.): Mapping Policy Preferences. Estimates for Parties, Electors and Governments 1945-1998. Oxford 2001; Hans-Dieter Klingemann / Andrea Volkens / Judith Bara / Ian Budge / Michael McDonald (Hrsg.): Mapping policy preferences II: Estimates for parties, electors and governments in Eastern Europe, European Union and OECD 1990-2003. Oxford 2006.

28 Hanna Bäck / Marc Debus / Patrick Dumont, »Who gets what in coalition governments? Predictors of portfolio allocation in parliamentary democracies « in: European Journal of Political Research 50 (2011) (im Erscheinen).

29 Vgl. etwa Wolfgang C. Müller / Kaare Strøm (Hrsg), Coalition Governments in Western Europe. Oxford 2000; Kaare Strøm / Wolfgang C. Müller / Torbjörn Bergman (Hrsg.), Delegation and Accountability in Parliamentary Democracies. Oxford 2003. 
rungsparteien, die nicht den Minister für einen bestimmten Politikbereich stellen, entsandt werden, um die Einhaltung des Koalitionsabkommens zu überwachen oder gar die vom Minister implementierte Policy in Richtung der eigenen Partei und deren Präferenzen abzuändern. ${ }^{30}$

Der Fokus auf die Ämterverteilung vernachlässigt jedoch die Bedeutung der in Deutschland seit der Bildung des sozialliberalen Kabinetts unter Bundeskanzler Helmut Schmidt (SPD) 1980 nach jeden Koalitionsverhandlungen geschlossenen Koalitionsabkommen, in denen neben der Festlegung der Ämtervergabe und der Formalia des Regierens in der Koalition vor allem die Politikziele der Bündnispartner für die kommende Legislaturperiode festgelegt werden. ${ }^{31}$ Während sich einige Arbeiten darauf konzentrieren, anhand der Regierungserklärung des Kanzlers oder Premierministers auf die künftige Politik der (Koalitions-)Regierung zu schließen, ${ }^{32}$ so sind zur Beantwortung der Frage, wie die künftigen Politikinhalte aussehen, Koalitionsabkommen überraschenderweise nur in geringem Ausmaß in empirische Analysen mit einbezogen worden.

In qualitativen Fallstudien zur Regierungsbildung und zum Regierungshandeln in den deutschen Bundesländern streichen Kropp und Sturm ${ }^{33}$ sowie Kropp ${ }^{34}$ die Bedeutung der Koalitionsabkommen gerade für das Verhalten von Landesregierungen im bikameralen politischen System der Bundesrepublik heraus. Timmermans verdeutlicht anhand von Fallstudien zum Handeln von Koalitionsregierungen in Belgien und den Niederlanden die Relevanz schriftlich fixierter Regierungsprogramme, die von allen Koalitionsparteien gemeinsam ausgehandelt werden. ${ }^{35}$ In empirisch-quantitativen Analysen, die auf einer Inhaltsanalyse der Regierungserklärungen und der Wahlprogramme der Regierungsparteien basieren, konnte Warwick zeigen, dass in der Tat eine große Übereinstimmung zwischen der erwarteten Position einer Koalition, gemessen auf Grundlage

30 Michael F. Thies, a.a.O. (FN 5); Luca Verzichelli, a.a.O. (FN 5).

$31 \mathrm{Vgl}$. Thomas Saalfeld, »Germany: Stable parties, chancellor democracy, and the art of informal settlement « in: Wolfgang C. Müller / Kaare Strøm (Hrsg), Coalition Governments in Western Europe. Oxford 2000; Arco Timmermans, »Standing Apart and Sitting Together: Enforcing Coalition Agreements in Multiparty Systems « in: European Journal of Political Research 45 (2006), H. 2, S. 263-283. Wolfgang C. Müller / Kaare Strøm, »Coalition Agreements and Cabinet Governance« in: Kaare Strøm / Wolfgang C. Müller / Torbjörn Bergman (Hrsg.): Cabinet Governance: Bargaining and the Cycle of Democratic Politics. Oxford 2008, S. 159-200.

32 Ian Budge / Michael Laver, »The Policy Basis of Government Coalitions: A Comparative Investigation « in: British Journal of Political Science 23 (1993), H. 4, S. 499-519; Thomas König / Andrea Volkens / Thomas Bräuninger, »Regierungserklärungen von 1949 bis 1998. Eine vergleichende Untersuchung ihrer regierungsinternen und -externen Bestimmungsfaktoren « in: Zeitschrift für Parlamentsfragen 30 (1999), H. 3, S. 641-659; Paul V. Warwick, »Coalition Policy in Parliamentary Democracies: Who Gets How Much and Why « in: Comparative Political Studies 34 (2001), H. 10, S. 1212-1236.

33 Sabine Kropp / Roland Sturm, Koalitionen und Koalitionsvereinbarungen: Theorie, Analyse und Dokumentation. Opladen 1998.

34 Sabine Kropp, Regieren in Koalitionen. Handlungsmuster und Entscheidungsbildung in deutschen Länderregierungen. Wiesbaden 2001.

35 Arco Timmermans, High Politics in the Low Countries: An Empirical Study of Coalition Agreements in Belgium and the Netherlands: Functions and Effects of Coalition Agreements in Belgium and The Netherlands. Aldershot 2003. 
der aus den Wahlprogrammen extrahierten Policy-Positionen, mit derjenigen der Regierungserklärung besteht. ${ }^{36}$ Dieser aus der Perspektive des »responsible party government «-Modells von Powell ${ }^{37}$ demokratietheoretisch positive Befund wird jedoch durch einen signifikanten Trend der Regierungserklärungen zu leicht rechteren Positionen auf einer ideologischen Links-Rechts-Achse getrübt. Auf Ebene der deutschen Bundesländer kommen Däubler und Debus in einer Analyse aller Wahlprogramme und Koalitionsabkommen zwischen 1990 und $2007 \mathrm{zu}$ einem ähnlichen Befund: die Regierungsparteien setzen zu einem großen Teil inhaltlich das im Koalitionsabkommen um, was sie auch in ihren Programmen während des Wahlkampfs geäußert haben. ${ }^{38}$ Jedoch ist auch hier eine signifikante Abweichung in sozioökonomischen Fragen zu mehr wirtschaftsliberalen Positionen feststellbar. Bevor auf den hier zu analysierenden Regierungsbildungsprozesses in Deutschland 2009 und dessen Resultate näher eingegangen wird, muss zunächst das bereits kurz vorgestellte »Political Heart «-Modell in der Form modifiziert werden, dass eine genaue Identifikation des zentralen Akteurs im Regierungsbildungsprozess möglich ist. Dies geschieht im folgenden Abschnitt.

\section{Theoretische Grundlagen zur Bestimmung pivotaler Akteure im Regierungsbildungsprozess}

Ein räumliches Modell wie das von Schofield entwickelte Konzept des »Political Heart « kann dazu herangezogen werden, um Aussagen zur parteipolitischen Zusammensetzung der Regierung einerseits wie auch der zentralen Akteure während der Koalitionsverhandlungen zu tätigen, da es sowohl die Stärke der Parteien im Parlament als auch deren programmatische Positionen auf zwei Politikfeldern berücksichtigt. Abbildung 1 veranschaulicht dies am Beispiel eines parlamentarischen Fünfparteiensystems. Parteien A und B erreichen in einem hypothetischen Parlament mit 100 Abgeordneten jeweils 40 Sitze, wohingegen Partei C auf 10 Mandate kommt. Parteien D und E stellen die beiden kleinsten Fraktionen mit jeweils 5 Sitzen dar. Aufgrund der Positionen der fünf im Parlament vertretenen Parteien entsteht nach dem Eintragen der Medianlinien ein »Political Heart «, das jeweils zwischen Mehrheit und Minderheit im zweidimensionalen Politikraum unterscheidet. Zentrale Akteure des Koalitionsspiels sind die Parteien A, B und C, wohingegen die beiden kleinen Parteien D und $\mathrm{E}$ als »peripher ${ }^{39}$ bezeichnet werden müssen, da durch ihre Position keine Medianlinie verläuft. Folgt man Schofields Modell, dann sollte die Regierung aus einer Koalition aus Parteien A und B, B und C oder A und $\mathrm{C}$ gebildet werden. Die Koalitionen $\mathrm{AC}$ und $\mathrm{BC}$ wären auf die Unterstützung einer der beiden kleinen Parteien angewiesen, da weder AC noch BC über die notwendige Mehr-

36 Paul V. Warwick, a.a.O. (FN 32).

37 G. Bingham Powell, Elections as Instruments of Democracy: Majoritarian and Proportional Visions. New Haven, London 2000; G. Bingham Powell, »The chain of responsiveness « in: Journal of Democracy 15 (2004), Heft 4, S. 91-105.

38 Thomas Däubler / Marc Debus, »Government Formation and Policy Formulation in the German States « in: Regional E Federal Studies 19 (2009), H. 1, S. 73-95.

39 Norman Schofield, a.a.O. (FN 2), S. 22. 
heit von 51 Sitzen im Parlament verfügen. Im Falle einer Regierung aus A und C wäre dies Partei E, deren Position rechts der Medianlinie AC liegt, während Partei D eine Koalition zwischen B und C unterstützen sollte, da diese den Policy-Präferenzen einer BC-Koalition am nächsten kommt.

Abbildung 1: "Political Heart«-Modell nach Schofield in einem bypothetischen Fünfparteiensystem

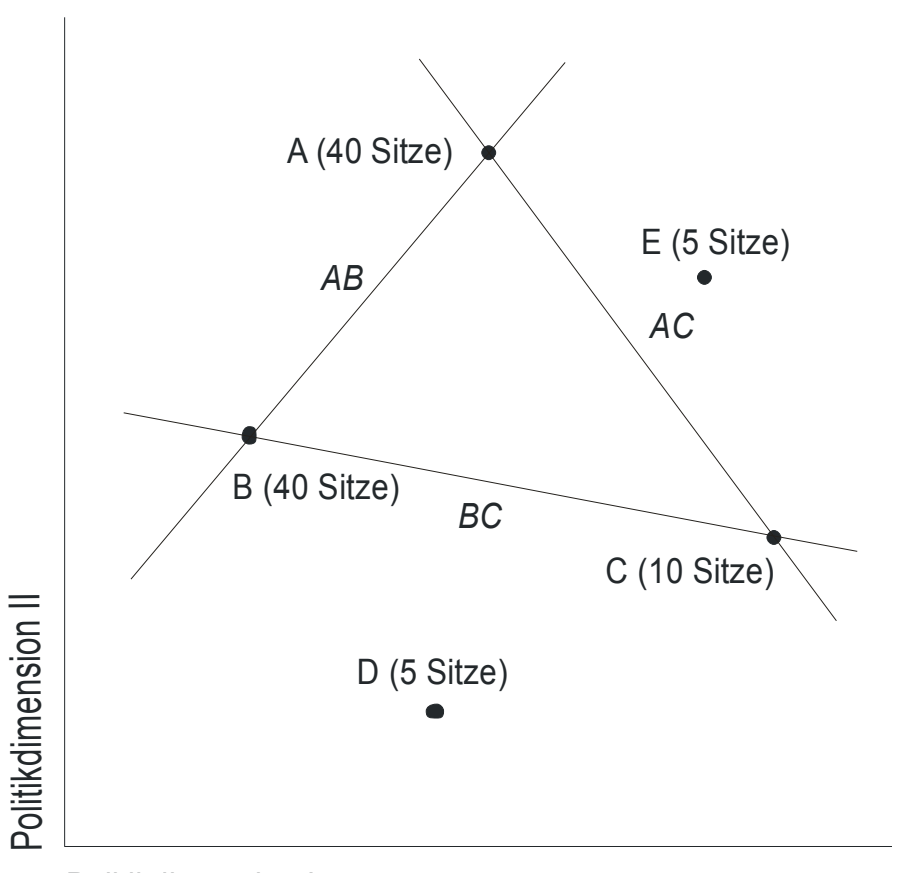

Politikdimension I

Dieses hypothetische Beispiel würde zwar eine Reihe von Erwartungen über die parteipolitische Zusammensetzung der künftigen Regierung liefern, kann jedoch keine präzise Aussage darüber machen, ob eine der fünf Parteien über eine besonders starke Position in den Koalitionsverhandlungen verfügen sollte. Dies wäre nur möglich, wenn alle Medianlinien durch die Position einer Partei verlaufen würden, welche dann zur »Core«Partei werden würde. Gemäß Schofields Modell sind in der Parteienkonstellation aus Abbildung 1 alle drei »Political Heart«-Parteien A, B und C die zentralen Akteure des Koalitionsbildungs- und Koalitionsverhandlungsprozesses. Bezieht man die Sitzstärke der Heart-Parteien mit ein, dann sollte die Position des Koalitionsabkommens als demjenigen Dokument, in dem die Politikziele der Regierung festgelegt werden, im Fall einer 
Koalition aus A und C sowie B und C jeweils näher an der stärkeren Partei und damit am programmatischen »centre of gravity « der Koalitionsregierung verortet sein. ${ }^{40}$

Eine solch eher unbefriedigende Vorhersage lässt sich jedoch beheben, wenn man Informationen zu kontextuellen Determinanten der Regierungsbildung in das räumliche Modell von Schofield $(1993,1995)$ mit einbezieht. Solche kontextuellen Ansätze thematisieren den Einfluss institutioneller Faktoren einerseits sowie »semi-institutioneller", politisch-kultureller Faktoren andererseits. ${ }^{41}$ Während ein Beispiel für den Einfluss von Institutionen auf die Muster der Regierungsbildung etwa in der Notwendigkeit von übergroßen Mehrheiten (z. B. zur Verfassungsänderung) liegen kann, so ist ein Beispiel für semi-instiutionelle Faktoren die Relevanz des Amtsinhaberstatus, ${ }^{42}$ die Kongruenz $\mathrm{zu}$ den Mustern von Regierung und Opposition auf anderen Ebenen des politischen Systems ${ }^{43}$ sowie die häufig vor Wahlen getätigten Koalitionsaussagen der Parteien. ${ }^{44}$ Koalitionsaussagen können positiv, aber auch negativ formuliert sein und üben einen eigenständigen Einfluss auf die parteipolitische Zusammensetzung einer Regierung aus. ${ }^{45}$ Gerade negativ formulierte Koalitionsaussagen haben in Form von Ausschlüssen diverser Koalitionsoptionen die Muster der Regierungsbildung in Deutschland und auf Ebene der Bundesländer maßgeblich geprägt. So hing das Scheitern des Regierungsbildungsprozesses nach den Landtagswahlen in Hessen vom Januar 2008 vor allem mit den von den Parteien a priori getätigten Koalitionsaussagen zusammen. So schloss die hessische SPD eine Koalition mit der CDU unter der Führung Roland Kochs aus, während die FDP die Bildung einer »Ampelkoalition« aus Sozialdemokraten, Grünen und Libe-

40 Donald A. Gross / Lee Sigelman, »Comparing party systems: A multidimensional approach in: Comparative Politics 16 (1984), H. 4, S. 463-479; vgl. Thomas R. Cusack, Partisan politics and public finance: Changes in public spending in the industrialized democracies, 19551989 « in: Public Choice 91, H. 3-4, S. 375-395; Thomas Bräuninger, »A Partisan Model of Government Expenditure« in: Public Choice 125 (2005), H. 3-4, S. 409-429.

41 Kaare Strøm / Ian Budge / Michael Laver, »Constraints on Cabinet Formation in Parliamentary Democracies « in: American Journal of Political Science 38 (1994), H. 2, S. 303-335; Sabine Kropp / Suzanne S. Schüttemeyer / Roland Sturm, »Koalitionen in West- und Osteuropa: Theoretische Überlegungen und Systematisierung des Vergleichs « in: Sabine Kropp / Suzanne S. Schüttemeyer / Roland Sturm (Hrsg.), Koalitionen in West- und Osteuropa. Opladen 2002, S. 7-41.

42 Hanna Bäck / Patrick Dumont, »Combining Large-n and Small-n Strategies: The Way Forward in Coalition Research« in: West European Politics 30 (2007), H. 3, S. 467-501.

43 Vgl. etwa Franz U. Pappi / Axel Becker / Alexander Herzog, »Regierungsbildung in Mehrebenensystemen: Zur Erklärung der Koalitionsbildung in den deutschen Bundesländern « in: Politische Vierteljabresschrift 46 (2005), H. 4, S. 432-458; Thomas Bräuninger / Marc Debus, a.a.O. (FN 4).

$44 \mathrm{Vgl}$. Sona Golder, »Pre-electoral coalitions in comparative perspective: A test of existing hypotheses « in: Electoral Studies 24 (2005), H. 4, S. 643-663; Sona Golder, »Pre-Electoral Coalition Formation in Parliamentary Democracies « in: British Journal of Political Science 36 (2006), H. 1, S. 193-212; Marc Debus, Pre-electoral alliances, coalition rejections, and multiparty governments. Baden-Baden 2007; Thomas Bräuninger / Marc Debus, a.a.O. (FN 4); Eric Linhart, a.a.O. (FN 4); Frank Decker, a.a.O. (FN 1).

45 Vgl. Thomas Bräuninger / Marc Debus, a.a.O. (FN 4); Marc Debus, a.a.O. (FN 4). 
ralen vorab verwarf. ${ }^{46}$ Eine Zusammenarbeit mit CDU und FDP wurde durch Bündnis 90/Die Grünen ausgeschlossen. Nachdem durch den Einzug der Linken in den Wiesbadener Landtag kein »traditioneller « Koalitionsblock - weder Schwarz-Gelb noch RotGrün - eine Mehrheit erreichte und die Bildung einer Minderheitsregierung aus SPD und Grünen unter der Tolerierung der Linken am Widerstand innerhalb der Sozialdemokraten scheiterte, waren Neuwahlen der einzige Ausweg aus der festgefahrenen Situation. ${ }^{47}$

Auf Bundesebene sah es im Vorfeld der Bundestagswahl 2009 zunächst so aus, als ob die Parteien aus der Gefahr zu starker Statements im Hinblick auf definitive Ausschlüsse diverser Koalitionsoptionen gelernt hätten und nur noch positiv formulierte Koalitionsaussagen und damit keine Koalitionsabsagen tätigen würden. Jedoch folgte dem Ausschluss einer Zusammenarbeit mit der Linken durch die SPD die Ablehnung der Grünen, sich an einem Bündnis mit CDU/CSU und FDP zu beteiligen. Auch die Liberalen haben schließlich mehrfach bekräftigt, keine Koalition mit Sozialdemokraten und Grünen eingehen zu wollen.

Baut man Informationen zu den seitens der Parteien vorab getätigten Koalitionsaussagen in das Modell des »Political Heart « ein, so kann dies entscheidende Verbesserungen in der Modellvorhersage nach sich ziehen. Abbildung 2 verdeutlicht diese Überlegung in graphischer Form: bezieht man zusätzlich zu der Stärke der Parteien und deren politikfeldspezifischen Positionen die Information mit ein, dass in dem bereits vorgestellten hypothetischen Fünfparteiensystem Parteien A und C eine Koalition a priori ausschließen, dann wird die zuvor bestehende und aus den Geraden AB, AC und BC begrenzte Zyklusmenge instabil. Die Ursache dafür ist, dass die Protokoalition AC nicht mehr als realistische Option aufgrund der Vorwahlstatements der Parteien gewertet werden kann. ${ }^{48}$ In Abbildung 2 ist dies durch die nunmehr nur noch gestrichelt eingezeichnete Mediangerade AC angedeutet. Die Folge ist, dass an der Position von Partei B ein »Core« entsteht, da sich dort die beiden verbliebenen Medianlinien schneiden. Aufgrund dieses durch die Berücksichtigung der Koalitionsaussagen modifizierten »Political Heart«Modells würde die Erwartung hinsichtlich der Regierungsbildung lauten, dass sich entweder eine Koalition zwischen den Parteien A und B oder B und C bilden würde. Damit würde das Modell auf der Analyseebene der Koalitionsbildung bereits eine genauere Vorhersage liefern. Deutlich präziser wäre hingegen die Vorhersage hinsichtlich der durchsetzungsfähigsten Partei. Im Beispiel aus Abbildung 2 sollte dies Partei B sein, da diese auf der Position des »Core « liegt und damit über ein Drohpotential in Koalitionsverhandlungen derart verfügt, dass man auch mit der jeweils anderen Protokoalitionspartei (in diesem Fall A oder C) ein Regierungsbündnis bilden könnte.

46 Vgl. Rüdiger Schmitt-Beck / Thorsten Faas, »Die hessische Landtagswahl vom 27. Januar 2008: Wiederkehr der >hessischen Verhältnisse« in: Zeitschrift für Parlamentsfragen 40 (2009), H. 1, S. 16-34.

47 Vgl. Rüdiger Schmitt-Beck / Thorsten Faas, a.a.O. (FN 46), S. 32.

48 Theoretisch betrachtet reicht es aus, wenn nur eine Partei der Protokoalition AC eine gemeinsame Regierung ausschließt, damit die Medianlinie nicht mehr gewertet werden kann. 
Abbildung 2: Um Koalitionsaussagen der Parteien erweitertes »Political Heart«-Modell in einem hypothetischen Fünfparteiensystem

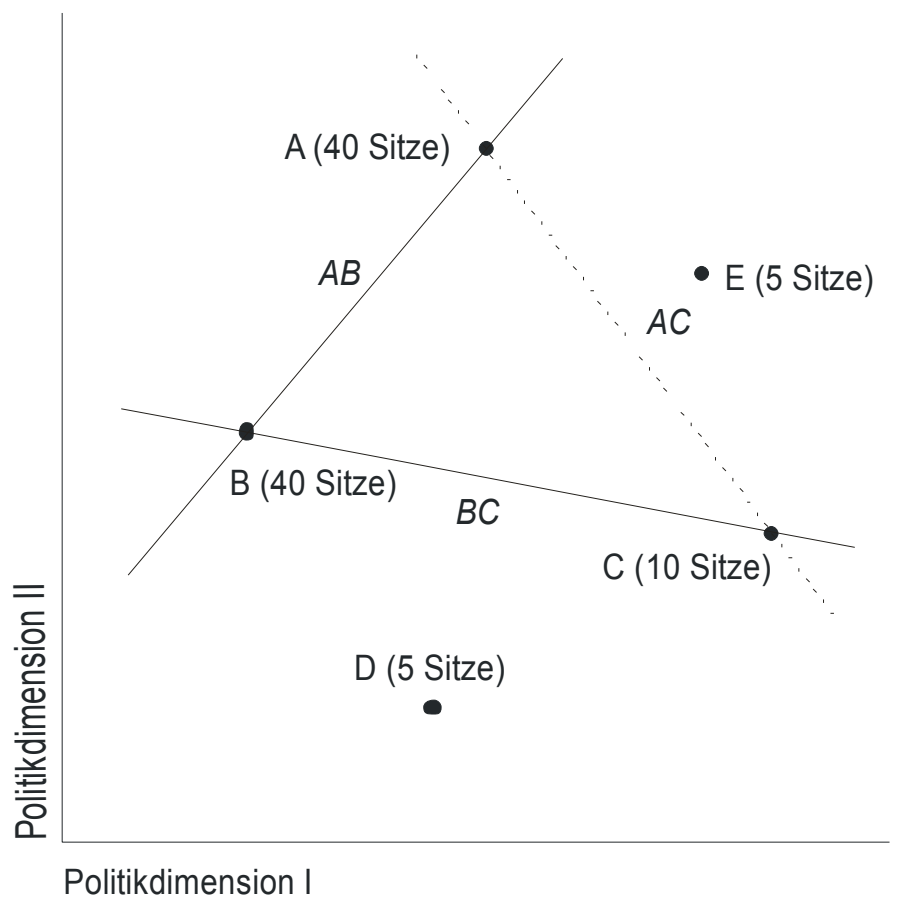

Zwar kann man argumentieren, dass Parteien mit Hinblick auf das Ziel der Regierungsbeteiligung ihren vor den Wahlen getätigten Koalitionsaussagen wenig Beachtung schenken. Jedoch zeigen zahlreiche Beispiele, dass ein solches Handeln das neben office- und policy-seeking dritte Ziel von Parteien - das Maximieren ihres Stimmenanteils - massiv gefährden kann. ${ }^{49}$ So verloren beispielsweise die Freien Demokraten nach ihren gebrochenen Koalitionsaussagen 1961, als sie eine Koalition mit CDU und CSU nur ohne einen Kanzler Adenauer eingehen wollten, und 1982, als die FDP in der Mitte der Legislaturperiode das sozialliberale Bündnis zu Gunsten einer Koalition mit der Union beendete, deutlich an Mitgliedern und Stimmen bei Bund- und Landtagswahlen. ${ }^{50}$ Das jüngste

49 Kaare Strøm / Wolfgang C. Müller, »Political Parties and Hard Choices« in: Wolfgang C. Müller / Kaare Strøm (Hrsg.): Policy, Office, or Votes? How Political Parties in Western Europe Make Hard Decisions. Cambridge 1999, S. 1-35.

50 Peter Lösche / Franz Walter, Die FDP. Richtungsstreit und Zukunftszweifel. Darmstadt 1996, S. 53-59; Thomas Poguntke, »Winner Takes All: The FDP in 1982-1983: Maximizing Votes, Office, and Policy? « in: Wolfgang C. Müller / Kaare Strøm (Hrsg.): Policy, Office, or Votes? How Political Parties in Western Europe Make Hard Decisions. Cambridge 1999, S. 216-236; Jürgen Dittberner, Die FDP: Geschichte, Personen, Organisation, Perspektiven. Eine Einführung. Wiesbaden 2005, S. 48-51. 
Beispiel, dass die elektoralen Konsequenzen von nicht eingehaltenen Koalitionsaussagen verdeutlicht, war die Strategie der hessischen Sozialdemokraten nach den Landtagswahlen 2008, als die dortige SPD trotz einer deutlichen Absage an eine Zusammenarbeit mit der Linken in Koalitionsverhandlungen mit der PDS-Nachfolgepartei eintrat, die dann aufgrund innerparteilicher Konflikte jedoch scheiterten. Die Neuwahlen zum hessischen Landtag vom Januar 2009 führten zu einem drastischen Rückgang des SPD-Stimmenanteils von 36,7 \% auf 23,7\%, der maßgeblich auf die gebrochene Koalitionsaussage der Sozialdemokraten um ihre damalige Vorsitzende und Spitzenkandidatin Andrea Ypsilanti zurückzuführen war. ${ }^{51}$

Aufbauend auf dieser Modifizierung des »Political Heart«-Modells von Schofield ${ }^{52}$ lässt sich folgende Hypothese formulieren: Wenn eine Partei Teil des um Informationen zu den Koalitionsaussagen erweiterten »Political Heart « ist, dann wird diese eine zentrale Stellung im Regierungsbildungsprozess einerseits und den Koalitionsverhandlungen andererseits einnehmen. Letzteres sollte dazu führen, dass sie mit Hinblick auf die Ämterverteilung (1) einen im Vergleich zur Stärke der Partei innerhalb der Koalitionsregierung überdurchschnittlich hohen Anteil an Ministerien für sich reklamieren kann und (2) im Vergleich zu den übrigen Koalitionspartnern mehr parlamentarische Staatssekretäre als »watch dogs« in "gegnerische«, also von den anderen Regierungsparteien kontrollierte Ministerien entsenden kann. Hinsichtlich der Politikziele der Regierungskoalition kann (3) erwartet werden, dass die Position des Koalitionsabkommens je Politikfeld im Vergleich zum Erwartungswert - dem mit der koalitionsinternen Stärke der Parteien gewichteten Mittelwert der Policy-Positionen aller Regierungsparteien - näher an der Position der Partei liegt, die Teil der modifizierten Zyklusmenge ist. Um diese Erwartungen am Beispiel der Regierungsbildung und Koalitionsverhandlungen nach der Bundestagswahl 2009 zu testen, bedarf es vor allem Daten zu den Policy-Positionen der politischen Akteure als auch zur Anzahl der in den Koalitionsverhandlungen gewonnenen politischen Ämter. Im nächsten Abschnitt wird dargelegt, auf welcher Grundlage und Methode die entsprechenden Daten ermittelt werden.

\section{Operationalisierung}

Zur Beantwortung der in diesem Beitrag aufgeworfenen Fragestellung sind zum einen Daten über die Anzahl der von den Regierungsparteien besetzten Ministerien und Staatsekretäre sowie Informationen zur Stärke der Parteien im Bundestag notwendig. Zum andern müssen die Policy-Positionen aller Bundestagsparteien auf den beiden für Deutschland zentralen Politikdimensionen ermittelt werden. Während Informationen zur Verteilung der Ämter auf die Regierungsparteien sowie über die Mitgliederzahl der Parlamentsfraktionen sehr leicht über die Internetauftritte der Bundesregierung und der

51 Vgl. Rüdiger Schmitt-Beck / Thorsten Faas, »Die hessische Landtagswahl vom 18. Januar 2009: der >ewige Koch« in: Zeitschrift für Parlamentsfragen 40, H. 2, S. 358-370.

52 Vgl. Norman Schofield, a.a.O. (FN 1); Marc Debus, »Office and Policy Payoffs in Coalition Governments« in: Party Politics 14 (2008), H. 5, S. 526-527. 
einzelnen Bundesministerien ${ }^{53}$ bzw. über die des Bundeswahlleiters ${ }^{54}$ zu bekommen sind, so wirft die Bestimmung der programmatischen Positionen der Parteien zur Wahl 2009 wie auch die Politikziele des CDU/CSU-FDP-Kabinetts größere Hürden auf. Eine Fortschreibung des vielfach zur Messung der programmatischen Schwerpunkte und Positionen von Parteien und Regierungen verwendeten CMP-Datensatzes liegt zwar vor, jedoch umfasst diese keine Informationen zu den in Koalitionsabkommen getätigten inhaltlichen Aussagen der jeweiligen Regierung. ${ }^{55}$ Daher wird hier auf das inzwischen vielfach erfolgreich angewandte, rein computergestützte »Wordscores «-Verfahren zur Messung der in Wahlprogrammen wie auch im Koalitionsabkommen enthaltenen Policy-Positionen zurückgegriffen. ${ }^{56}$ Das Verfahren geht von der Beobachtung aus, dass die Wortwahl politischer Akteure in ihren programmatischen Dokumenten oder Reden nicht nach dem Zufallsprinzip erfolgt. Vielmehr senden beispielsweise Parteien in ihren Wahlprogrammen »ideologische Signale «, 57 um ihre Anhängerschaft vor der Wahl zu mobilisieren. Daher gehen Laver, Benoit und Garry 58 von der Annahme aus, dass man allein aufgrund der relativen Worthäufigkeit eines Dokuments auf dessen programmatische Position schließen kann. Das Grundprinzip von »Wordscore « ist daher, die relative Worthäufigkeit eines Textes, dessen Position man kennt (so genannte »Referenztexte«), mit der relativen Häufigkeit jedes Wortes eines unbekannten Textes zu vergleichen. Da die Position des Referenztexts bekannt ist, kann auf die Position des neuen Textes geschlossen werden. Als Referenzwerte können beispielsweise Ergebnisse von Expertenbefragungen dienen.

Einem auf der Grundlage dieses Verfahrens bereits erstellten Datensatzes, der die Positionen der deutschen Bundes- und Landesparteien seit 1990 umfasst, ${ }^{59}$ wurden die zu den Bundestagswahlen 2009 verfassten programmatischen Dokumente inklusive des Koalitionsabkommens hinzugefügt. Damit ist es auch möglich, potentielle Veränderungen in den Positionen der Parteien zu analysieren. Im folgenden Abschnitt werden die Erwartungen hinsichtlich des Durchsetzungsvermögens der Parteien - differenziert nach Ämterverteilung und Politikzielen - anhand der hier verwendeten Daten überprüft.

$53 \mathrm{Vgl.} \mathrm{http://www.bundesregierung.de/Webs/Breg/DE/Bundesregierung/Bundeskabinett/bun}$ deskabinett.html.

54 Vgl. http://www.bundeswahlleiter.de/de / bundestagswahlen / BTW_BUND_09/ ergebnisse/ bundesergebnisse/grafik_sitze_99.html.

55 Vgl. Ian Budge / Hans-Dieter Klingemann / Andrea Volkens / Judith Bara / Eric Tanenbaum, a.a.O. (FN 27); Hans-Dieter Klingemann / Andrea Volkens / Judith Bara / Ian Budge / Michael McDonald, a.a.O. (FN 27).

56 Michael Laver/Kenneth Benoit/John Garry,»Extracting Policy Positions from Political Texts Using Words as Data in: American Political Science Review 97 (2003), H. 2, S. 311-331; Will Lowe, »Understanding Wordscores« in: Political Analysis 16 (2008), H. 4, 356-371.

57 Franz U. Pappi / Susumu Shikano, Ideologische Signale in den Wahlprogrammen der deutschen Bundestagsparteien 1980 bis 2002. MZES Working Papers Nr. 76. Mannheim 2004.

58 Michael Laver / Kenneth Benoit / John Garry, a.a.O. (FN 56).

59 Thomas Bräuninger / Marc Debus, a.a.O. (FN 4); Thomas Bräuninger / Marc Debus, Parteienwettbewerb in den deutschen Bundesländern. Wiesbaden 2010 (in Vorbereitung). 


\section{Analyse: Ämterverteilung und Politikziele der schwarz-gelben Koalitionsregierung}

Zunächst gilt es, diejenige der fünf im Bundestag vertretenen Parteien zu bestimmen, die Teil der gemäß der um die Koalitionsaussagen der Parteien erweiterten modifizierten »Political Heart «-Lösung sind. Abbildung 3 macht deutlich, dass drei Medianlinien, die zwischen Mehrheit und Minderheit im Bundestag trennen, das »Political Heart« begrenzen. Dies ist erstens die zwischen Union und FDP verlaufende Medianlinie, zweitens eine Gerade, deren Lage durch die Positionen von SPD und Liberalen festgelegt wird, und schließlich eine Mediangerade, die durch die wirtschafts- und gesellschaftspolitischen Positionen von CDU/CSU und SPD verläuft..$^{60}$ Somit ergibt die unmodifizierte Zyklusmenge, die Union, Sozialdemokraten und FDP umfasst, drei zentrale Akteure im Koalitionsspiel, die sich gemäß des »Political Heart«-Modells in drei Protokoalitionen übersetzen lassen. Dies ist erstens das Bündnis aus Christ- und Freidemokraten, das sich schließlich auch als Regierungskoalition im Oktober 2009 zusammengefunden hat, zweitens die zum Zeitpunkt der Wahl amtierende Koalition aus CDU/CSU und SPD und drittens eine Minderheitskoalition aus Sozialdemokraten und FDP, die mit der Unterstützung einer der beiden »peripheren « Parteien - den Bündnisgrünen oder der Linken - eine Mehrheit im Bundestag erreicht hätte.

Nun haben aber die Liberalen auf einem Parteitag kurz vor der Bundestagswahl eine Koalition mit SPD und Bündnis 90/Die Grünen ausgeschlossen. Ein Zusammengehen mit der Linkspartei - in welcher Parteienkombination auch immer - stand für die FDP ohnehin nicht zur Debatte. Berücksichtigt man diese Information bei der Erstellung des "Political Hearts «, dann darf man die Medianlinie, die zwischen Sozial- und Freidemokraten verläuft, nicht berücksichtigen, da eine Mehrheit unterhalb dieser Geraden und somit eine Koalition aus SPD, FDP und Grünen bzw. Sozialdemokraten, Freidemokraten und Linken seitens der Liberalen a priori ausgeschlossen wurde. Diese Zusatzinformation, die durch die gepunktet eingezeichnete Medianlinie zwischen SPD und FDP in Abbildung 3 kenntlich gemacht ist, hat einen bedeutenden Einfluss auf die Vorhersagekraft des Modells. So ergeben sich nunmehr lediglich zwei Koalitionsoptionen. Dies ist zum einen ein Bündnis aus Union und FDP und damit der Koalition, die beide Parteien aufgrund ihrer im Wahlkampf getätigten Koalitionsaussagen präferierten, sowie eine Neuauflage der zum Zeitpunkt der Bundestagswahl 2009 amtierenden Koalition aus CDU/CSU und SPD. Für die in diesem Beitrag behandelte Fragestellung ist von noch größerer Bedeutung, dass das für die Bundestagswahlen 2009 modifizierte »Political Heart«-Modell statt drei nur noch eine Partei als zentralen Akteur identifiziert. Dies rührt daher, dass aufgrund des de facto Wegfalls der durch die Positionen von SPD und FDP verlaufenden Mediangeraden ein »Core « an der Position der Unionsparteien entsteht. Als Kernpartei ist die CDU/CSU damit der zentrale Akteur im Regierungsbildungsprozess sowie bei den Koalitionsverhandlungen nach den Bundestagswahlen 2009.

60 Die Konfidenzintervalle der mit »Wordscores« geschätzten Policy-Positionen der Parteien werden bei der Ermittlung des Verlaufs der Mediangeraden nicht berücksichtigt. 
Abbildung 3: Modifiziertes "Political Heart zur Bundestagswabl 2009 auf Grundlage der Stärke der Fraktionen, Parteipositionen und Koalitionsaussagen

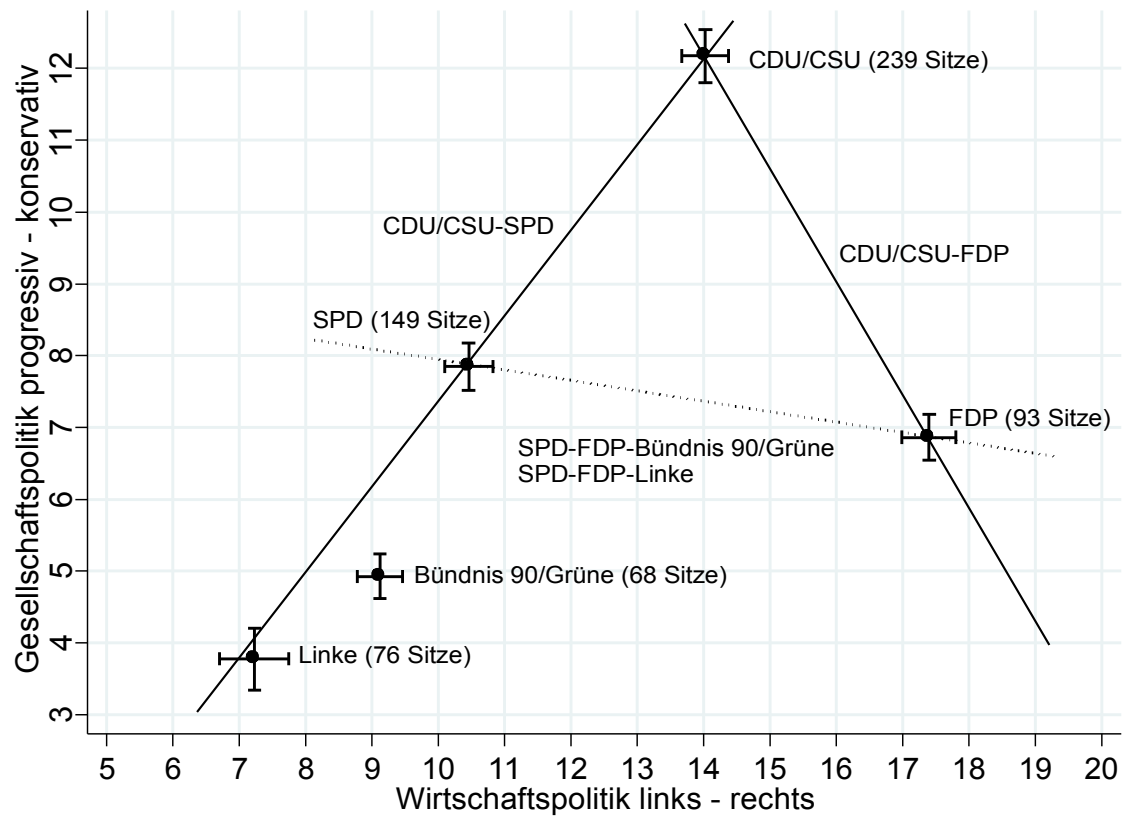

Bevor auf die Evaluierung der Durchsetzungsfähigkeit der Union bei den Koalitionsverhandlungen mit der FDP näher eingegangen wird, soll ein Vergleich zur Konstellation nach der Bundestagswahl 2005 deutlich machen, in welchem Ausmaß sich die Muster von Parteienwettbewerb und Regierungsbildung geändert haben. Aus den programmatischen Positionen und der Sitzstärke der Parteien im 16. Deutschen Bundestag hatte sich ein »Political Heart « aus Union, SPD und Bündnis 90/Die Grünen gebildet, womit FDP und PDS/Linke periphere Parteien waren (vgl. Abbildung 4). ${ }^{61}$ Aufgrund der Koalitionsaussagen der Parteien sind die sich aus der »Political Heart«-Lösung ergebenden Protokoalitionen aus CDU/CSU und Grünen einerseits sowie aus SPD, Bündnis 90/ Grüne und PDS/Linke andererseits auszuschließen. ${ }^{62}$ Die einzig übrig bleibende Medianlinie und Koalitionsoption - ein Bündnis aus Union und Sozialdemokraten - bildete schließlich auch die Bundesregierung. Die große Koalition aus CDU/CSU und SPD hatte 2005 zudem keine allzu großen inhaltlichen Konflikte zu überwinden: die inhalt-

61 Für ähnliche Resultate vgl. Franz U. Pappi / Susumu Shikano, »Regierungsabwahl ohne Regierungsneuwahl? « in: Politische Vierteljabresschrift 46 (2005), H. 4, S. 513-526; Franz U. Pappi, a.a.O. (FN 3).

62 Marc Debus, a.a.O. (FN 44), S. 46; Eric Linhart, a.a.O. (FN 4), S. 473, Frank Decker, a.a.O. (FN 1), S. 435. 
lich-programmatischen Distanzen zwischen den beiden großen Parteien waren - verglichen mit den Abständen zwischen Union und FDP oder SPD und Bündnis 90/Die Grünen - eher gering. Vergleicht man die Positionen der Parteien von 2005 mit denjenigen von 2009, die in Abbildung 3 abgetragen sind, dann zeigt sich, dass insbesondere die Sozialdemokraten zur Bundestagswahl 2009 eine gesellschaftspolitisch progressivere Position eingenommen und damit die Distanz zum Koalitionspartner CDU/CSU, der seine Positionen auf beiden hier untersuchten Dimensionen nahezu nicht verändert hat, vergrößert haben. Während auch die Liberalen sich gesellschaftspolitisch weiter in die progressive Richtung entwickelt haben, so haben sich die Positionen der beiden anderen Parteien Bündnis 90/Die Grünen und Linke zwischen 2005 und 2009 kaum verändert.

Abbildung 4: Modifiziertes "Political Heart " zur Bundestagswabl 2005 auf Grundlage der Stärke der Fraktionen, Parteipositionen und Koalitionsaussagen

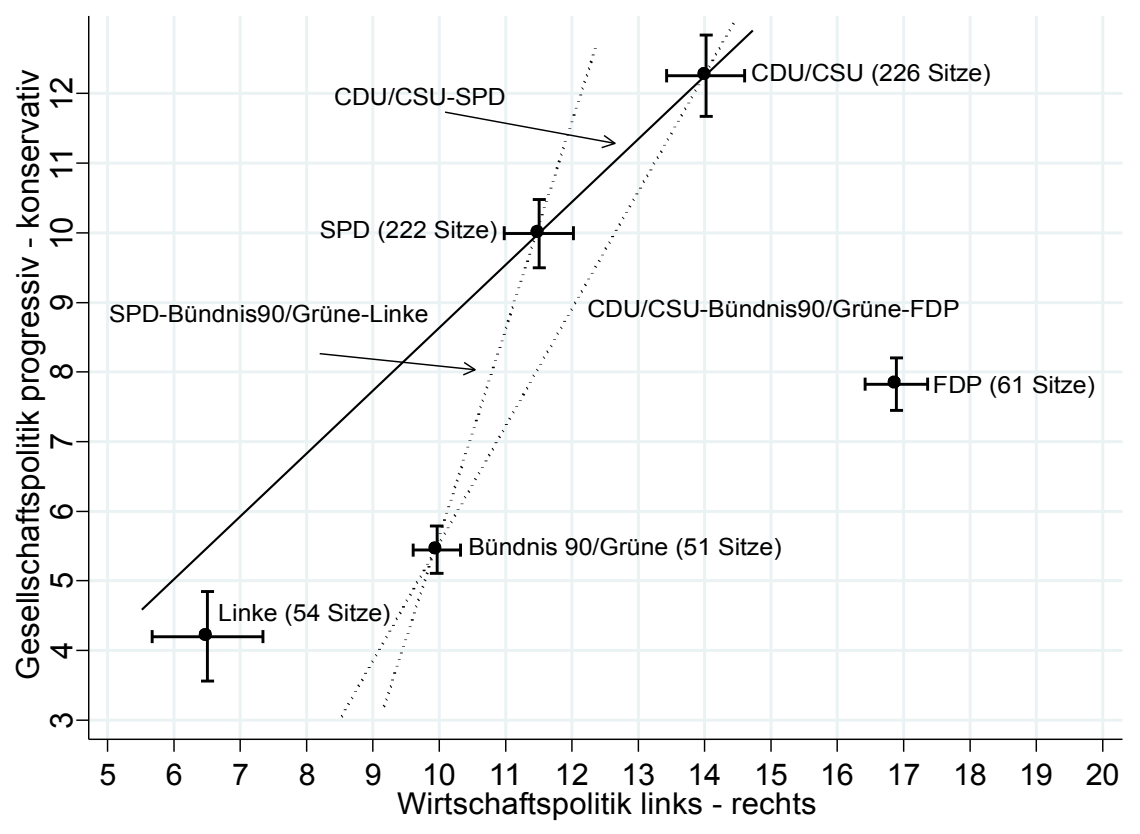

Um zu testen, ob sich die aufgrund des modifizierten »Political Heart«-Modells als Kernpartei definierte CDU/CSU sich überdurchschnittlich stark in den Koalitionsverhandlungen hat durchsetzen können, müssen die Erwartungswerte hinsichtlich der Verteilung der Kabinettsposten sowie der inhaltlichen Positionen der neuen Koalitionsregierung mit den gemessenen Werten abgeglichen werden. Mit Rückgriff auf die Proportionalitätsnorm sollte man erwarten, dass die Ämter in dem Verhältnis auf die Regierungsparteien verteilt werden, in welchem diese zur Mehrheit der Koalition im Bundestag beitragen. Gleiches sollte für die Policy-Position des Koalitionsabkommens von 
Union und FDP gelten: es sollte an dem mit der Stärke der Regierungsparteien gewichteten arithmetischen Mittel - dem programmatischen »centre of gravity «63 - im Politikraum verortet sein.

Tabelle 1 gibt den Anteil der Sitze wieder, die die Regierungsparteien CDU/CSU64 und FDP zur Anzahl der Mandate der Regierungskoalition beitragen, sowie den Anteil der gewonnenen Ministerposten - jeweils ungewichtet als mit der Bedeutung der Ministerien gewichtet - und derjenigen der parlamentarischen Staatssekretäre, die zur Union bzw. den Liberalen gehören. Zusätzlich dazu wird die Anzahl der Ministerien angegeben, in denen es den beiden Regierungsparteien gelang, einen Staatssekretär in einem vom Koalitionspartner kontrollierten Ministerium zu entsenden, so dass dieser als »watch dog « im Sinne von Thies gelten kann. ${ }^{65}$ Gemäß den in Tabelle 1 präsentierten Daten konnte die gemäß des modifizierten Political Heart-Modells identifizierte »Kernpartei« CDU/CSU in Fragen der Ministerienverteilung nicht von ihrer pivotalen Rolle im Koalitionsspiel profitieren. Vielmehr konnten die Freidemokraten einen höheren Anteil an Ministerien für sich reklamieren (33,3\%), als ihnen anhand ihrer Stärke innerhalb der Koalitionsregierung - die FDP-Abgeordneten machen $28 \%$ der gemeinsamen Bundestagsmehrheit mit der Union aus - zugestanden hätte. Dies gilt auch, wenn man das Amt der Bundeskanzlerin in die Analyse mit einbezieht. Da auch die CSU - im Vergleich zu ihrem Mandatsanteil innerhalb der Koalition - überdurchschnittlich viele Ministerien für sich reklamieren konnte, wird der von Browne und Franklin beobachtete Vorteil für die kleineren Koalitionsparteien bei der Ämterverteilung auch bei der Regierungsbildung in Deutschland 2009 erneut deutlich. ${ }^{66}$

Diese Perspektive verschiebt sich nur geringfügig in Richtung der »core party « CDU/ CSU, wenn die Ministeriumsverteilung mit der Salienz der jeweiligen Ministerien anhand der Daten von Druckman und Warwick gewichtet wird. ${ }^{67}$ Zwar gleicht sich die Ministerienverteilung der Proportionalitätsnorm stärker an, jedoch bleiben die Unionsparteien noch immer leicht hinter dem Erwartungswert zurück. Dies gilt wiederum auch, wenn das Amt der Kanzlerin mit einbezogen wird. Das unter Berücksichtigung der Ministe-

63 Donald A. Gross / Lee Sigelman, a.a.O. (FN 40).

64 CDU/CSU werden in der Analyse als einheitlich handelnde Partei ausgewiesen, da sie im Bundestag eine Fraktionsgemeinschaft bilden und auch ein gemeinsames Wahlprogramm verfasst haben. Der Vollständigkeit halber wird in Tabelle 1 bei der Analyse der Kongruenz von Sitzanteilen und der Anzahl der gewonnenen Ministerien und Staatssekretäre zwischen CDU und CSU differenziert.

65 Martin F. Thies, a.a.O. (FN 5); Luca Verzichelli, a.a.O. (FN 5), S. 259-264.

66 Eric C. Browne / Mark N. Franklin, a.a.O. (FN 21).

67 In der Expertenbefragung wurde erhoben, welche Ministerien in 14 westeuropäischen Demokratien welchen Grad an Bedeutung haben. Wenn die Befragten einem Ministerium einen Wert von » 1 « zuordneten, dann handelte es sich um ein durchschnittlich-wichtiges Ministerium. Ein Wert von z.B. »1,5« sollte vergeben werden, wenn das entsprechende Ministerium um $50 \%$ wichtiger eingestuft wird als ein durchschnittlich wichtiges Ministerium. Dementsprechend sollte ein Wert von unter $» 1$ «vergeben werden, wenn es sich um ein unterdurchschnittlich wichtiges Ministerium handelt. Vgl. James N. Druckman / Paul V. Warwick, The missing piece: Measuring portfolio salience in Western European parliamentary democracies, in: European Journal of Political Research 44 (2005), H. 1, S. 17-42. 
riumssalienzen die Union - und hier ausschließlich die CDU - ihre unterdurchschnittlichen Payoffs leicht ausgleichen kann, liegt darin begründet, dass sie gemäß den Resultaten von Druckman und Warwick die eher wichtigen Ministerien besetzen konnte, wohingegen nur zwei der fünf Ministerien, die an die FDP gingen, als überdurchschnittlich "salient " gelten. Dies sind das mit dem FDP-Vorsitzenden Guido Westerwelle besetzte Außenministerium, das einen Wert von 1,41 in der Studie von Druckman und Warwick erreicht, sowie das Justizministerium, dem die Liberale Sabine Leutheusser-Schnarrenberger vorsteht und das auf einen geringfügig überdurchschnittlichen Wert von 1,02 kommt. $^{68}$

68 Vgl. James N. Druckman / Paul V. Warwick, a.a.O. (FN 67), S. 35-36.

ZfP 57. Jg. $4 / 2010$ 


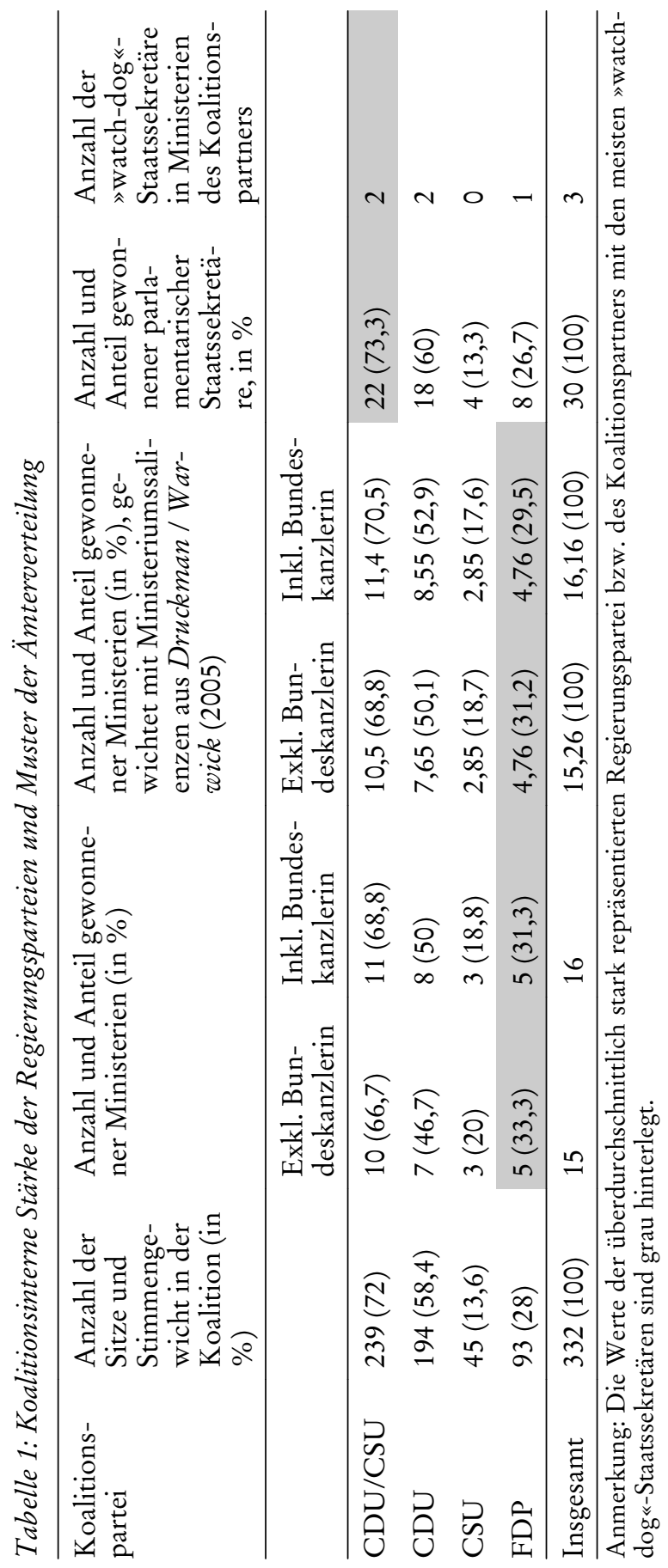


Erst wenn die parlamentarischen Staatssekretäre und ihre potentielle Verwendung als »watch-dogs « in die Analyse mit einbezogen werden, dann äußert sich partiell die Verhandlungsstärke der Union hinsichtlich der Ämterverteilung: so gehören 22 von 30 und damit 73,3 \% der parlamentarischen Staatssekretäre der CDU/CSU an. Die Freien Demokraten erreichen mit acht Staatssekretären einen leicht unterdurchschnittlichen Anteil an allen parlamentarischen Staatssekretärsposten (26,7\%). Deutlicher wird die zentrale Position der Union in der koalitionsinternen Ämtervergabe, wenn man sich die Anzahl der Ministerien anschaut, in dem die Union einen Staatssekretärsposten besetzen kann, obwohl die FDP den jeweiligen Minister stellt. Dies ist der Fall in dem von Rainer Brüderle (FDP) geleiteten Ministerium für Wirtschaft und Technologie sowie im vom Freidemokraten Philipp Rösler geführten Gesundheitsministerium, wo CDU und Liberale jeweils einen Staatsekretär stellen. Im Gegenzug konnte die FDP nur einen » watch-dog « in Form eines parlamentarischen Staatssekretärs in einem von der Union geführten Ministerium platzieren: dies gelang den Liberalen im Verkehrs-, Bau- und Stadtentwicklungsministerium von Peter Raumsauer (CSU), wo gleich drei Staatssekretärsposten, die von CDU, CSU und FDP besetzt wurden, geschaffen worden sind. Vergleicht man diese Muster der Ämterverteilung auf Minister- wie Staatssekretärsebene mit dem Kabinett der großen Koalition unter Kanzlerin Merkel von 2005 bis 2009, so fällt auf, dass in der Vorgängerregierung weder die Union noch die SPD Staatssekretärsposten in vom Koalitionspartner geführte Ministerien entsenden konnten. ${ }^{69}$ Dies kann auch damit erklärt werden, dass CDU und CSU 2005 nicht die Eigenschaften einer »core party « besaßen (vgl. Abbildung 4). Insofern kann argumentiert werden, dass aufgrund der Mandatsstärke, der politikfeldspezifischen Positionierung und der Koalitionsaussagen der im Bundestag vertretenen Parteien gewonnene zentrale Position der Union im Koalitionsspiel zu einer für sie günstigeren Ämterverteilung zumindest hinsichtlich der Besetzung von parlamentarischen Staatssekretären allgemein und von »watch-dogs « im Besonderen geführt hat.

Zeigt sich ein klareres Bild hinsichtlich des erwarteten Durchsetzungsvermögens der CDU/CSU, wenn man statt der Ämterverteilung den Blick auf die inhaltlichen Positionen des Koalitionsabkommens richtet? Abbildung 5 veranschaulicht das Ergebnis in grafischer Form. Die mit Hilfe des »Wordscores«-Verfahrens gemessene Position des Koalitionsabkommens von Union und Liberalen liegt - selbst unter Berücksichtigung des geschätzten 95 \%-Konfidenzintervalls, dessen Größe anhand von Balken durch den Idealpunkt des Koalitionsabkommens wie auch der Parteipositionen eingezeichnet ist nicht am Gravitätszentrum der schwarz-gelben Bundesregierung im hier aufgespannten wirtschafts- und gesellschaftspolitischen Politikraum. Vielmehr zeigt die ermittelte Position des Koalitionsabkommens, dass sich auf der sozioökonomischen Links-RechtsDimension CDU und CSU zumindest so gut wie erwartet durchsetzen konnten, da sich die erwartete sozioökonomische Position der Koalition mit der ermittelten weitgehend

69 Vgl. Richard Hilmer / Rita Müller-Hilmer, »Die Bundestagswahl vom 18. September 2005: Votum für Wechsel in Kontinuität« in: Zeitschrift für Parlamentsfragen 37 (2006). H. 1, S. 214-215; Marc Debus, a.a.O. (FN 44), S. 51. 
deckt. Damit konnte sich die FDP auf dem für sie und ihre Anhängerschaft zentralen Politikfeld der Wirtschafts- und Sozialpolitik nicht überdurchschnittlich stark durchsetzen. Dies gelang den Liberalen jedoch in Fragen der Innen-, Rechts- und Gesellschaftspolitik: hier nimmt das Koalitionsabkommen des Kabinetts Merkel/Westerwelle eine im Vergleich zum Erwartungswert signifikant progressivere Position ein, die deutlich näher an der Position der FDP als an derjenigen der Christdemokraten und Christsozialen liegt.

Abbildung 5: Erwartete und tatsächliche Policy-Position des schwarz-gelben Koalitionsabkommens $2009 \mathrm{im}$ Politikraum

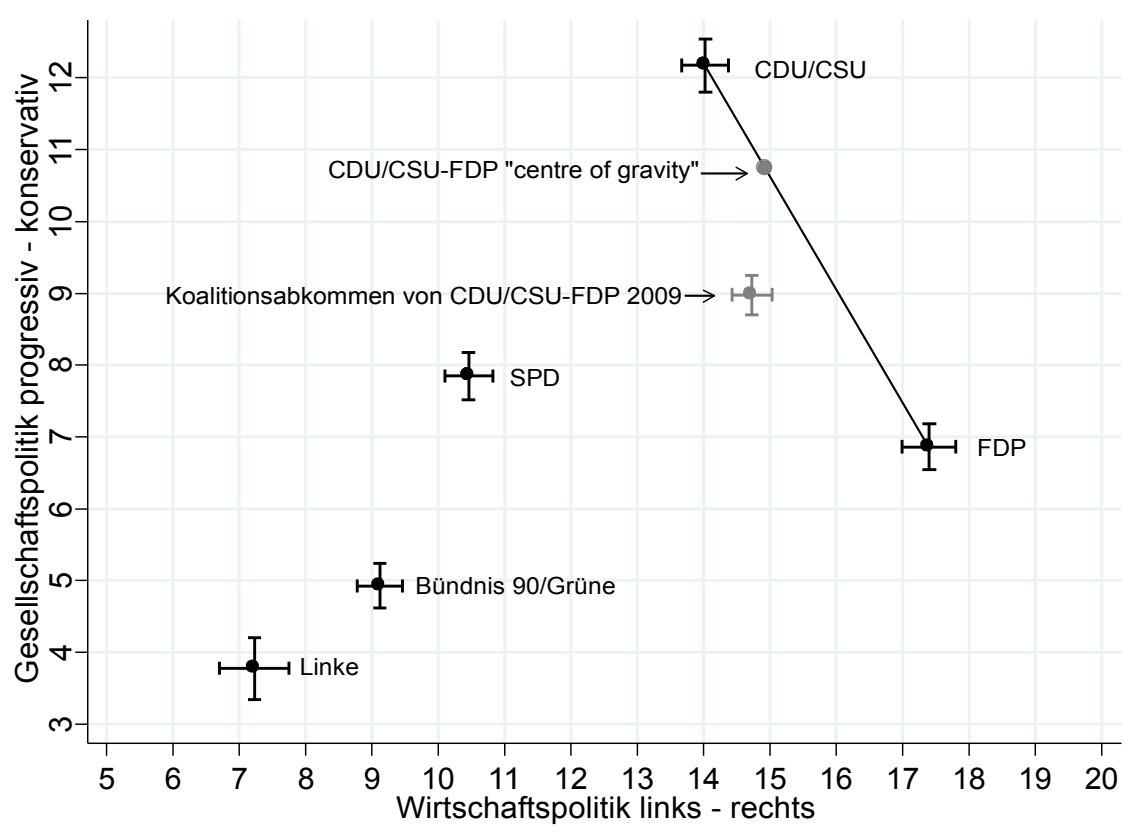

\section{Schlussbetracbtung}

Die Kombination »klassischer « koalitionstheoretischer Ansätze, die sich auf die Prinzipien der Ämtergewinnung und programmatischen Nähe politischer Akteure beziehen, ermöglichen zusammen mit kontextuellen Erklärungsfaktoren ein detailliertes Bild des Koalitionsbildungs- und Regierungsformierungsprozesses. Wie am Beispiel des »Political Heart«-Modells gezeigt werden konnte, liefert die Einbeziehung von a priori abgelehnten Koalitionsoptionen entscheidende Hinweise darauf, welche der im Parlament vertretenen Parteien der zentrale Akteur im Koalitionsspiel nach einer Wahl ist. Die Anwendung dieses Modells auf den Regierungsbildungsprozess in Deutschland nach der 
Bundestagswahl 2009 wies die Unionsparteien als modifizierte »Kernpartei« aus, was ihr theoretisch betrachtet ein hohes Durchsetzungsvermögen in den Verhandlungen mit dem von ihr präferierten Regierungspartner FDP hätte gewährleisten sollen. Die genauere Betrachtung der Ministerienverteilung hat jedoch deutlich gemacht, dass auf dieser Ebene vielmehr die Liberalen und nicht die CDU/CSU einen überdurchschnittlichen Anteil an Ministerien erringen konnten. Dies gilt jedoch nicht für die parlamentarischen Staatssekretäre: diese konnte die Union doppelt so oft wie die FDP in Form von »PolicyKontrolleuren « in von liberalen Politikern kontrollierte Ministerien entsenden.

Die Inhaltsanalyse der Bundestagswahlprogramme der Parteien sowie des Koalitionsabkommens 2009 hat des weiteren deutlich gemacht, dass sich insbesondere die Position der Sozialdemokraten im Vergleich zu ihrem Wahlmanifest 2005 gesellschaftspolitisch deutlich in die progressive Richtung verschoben hat, so dass ein Bündnis CDU/ CSU-SPD 2009 weit weniger programmatisch kohäsiv war als noch nach der Bundestagswahl vom September 2005. Im Hinblick auf die inhaltlichen Kompromisse der Regierungskoalition, festgehalten im Koalitionsabkommen, konnten sich zwar die Liberalen in gesellschaftspolitischen Fragen verhältnismäßig stark mit ihren Vorstellungen durchsetzen, nicht jedoch im sozioökonomischen Bereich, wo die Position des Regierungsprogramms deutlich näher an der Position des CDU/CSU-Wahlprogramms liegt.

Diese Resultate machen deutlich, dass ein hohes Durchsetzungsvermögen bei der Ämterverteilung in Koalitionsregierungen nicht unbedingt einhergeht mit den inhaltlichen Kompromissen, auf die sich die Regierungsparteien geeinigt haben. Auch scheint den parlamentarischen Staatssekretären eine besondere Rolle bei der koalitionsinternen Form von »checks and balances « zuzukommen. Hieraus lässt sich ein Anreiz für weitere Studien zum Regieren in Koalitionen ableiten, die tief greifender mit Hilfe qualitativer Fallstudien auf den Zusammenhang zwischen Ämterverteilung und den Inhalten des Koalitionsabkommens eingeht. ${ }^{70}$ Zudem steht zu überprüfen, inwiefern die hier anhand des Regierungsprogramms gemessenen Policy-Positionen der schwarz-gelben Bundesregierung Merkel/Westerwelle auch tatsächlich in Policy Outputs umgesetzt werden. ${ }^{71}$ Dies kann - wie auch die detaillierte Analyse der Determinanten von positiv wie negativen Koalitionsaussagen im deutschen wie internationalen Kontext - ebenfalls Aufgabe künftiger Forschungsarbeit sein.

\section{Zusammenfassung}

Neben der Frage nach den Determinanten der parteipolitischen Zusammensetzung ist die Aufteilung der Ämter sowie die inhaltlich-programmatischen Kompromisse ein zentraler Aspekt der Analyse von Koalitionsregierungen. In diesem Aufsatz wird auf

70 Vgl. für die Koalitionsverhandlungen nach der Bundestagswahl 2009 etwa Thomas Saalfeld, »Regierungsbildung 2009: Merkel II und ein höchst unvollständiger Koalitionsvertrag « in: Zeitschrift für Parlamentsfragen 41 (2010). H. 1, S. 181-206.

71 Vgl. für eine Evaluierung der Politik des zweiten Schröder-Kabinetts Christoph Egle / Reimut Zohlnhöfer (Hrsg.), Ende des rot-grünen Projektes. Eine Bilanz der Regierung Schröder 2002-2005. Wiesbaden 2007. 
Grundlage der Verteilung der Kabinettspositionen sowie der im Koalitionsabkommen formulierten künftigen Politikziele untersucht, ob sich CDU/CSU oder FDP in den Koalitionsverhandlungen 2009 besser durchsetzen konnten. Auf Grundlage des um Informationen zu den Koalitionsaussagen der Parteien erweiterten Modells des »Political Heart « kann die Union als der entscheidende Akteur im Koalitionsbildungs- und verhandlungsprozess 2009 bestimmt werden. Jedoch konnte sich die Union nicht in dem Maße durchsetzen, wie es aufgrund der Modellvorrausage erwartet wurde: im Vergleich zu den in den Wahlprogrammen geäußerten programmatischen Positionen konnte die CDU/CSU ihre Vorstellungen vor allem in wirtschafts- und sozialpolitischen Fragen in das Koalitionsabkommen einbauen, während dies der FDP im Bereich Gesellschaftspolitik gelang. Hinsichtlich der Ämterverteilung erreichte die Union keinen überdurchschnittlichen Ministeranteil, wohl aber einen in der Gruppe der parlamentarischen Staatssekretäre, die die Christdemokraten zudem in zwei von der FDP kontrollierten Ministerien platzieren konnten.

\section{Summary}

The question »who gets what? « is one of the central research areas in studies on coalition politics. This paper analyses which party of the new German coalition government between Christian Democrats and Liberals was able to implement its office and policy preferences at most. On the basis of the »Political Heart« model, which is modified in a way that pre-electoral coalition statements of the parties are included, the paper argues that CDU/CSU should be in a pivotal position in the 2009 German coalition formation game as well as in the coalition negotiation process. When evaluating this expectation empirically, there is, however, only mixed evidence for this expectation. CDU and CSU were only able to implement their economic policy positions, but not their societal policy preferences in the negotiated policy agreement. In addition, they did not receive a surpassing share of cabinet portfolios. Yet, Christian Democrats could win a larger number of junior ministers and were additionally able to place two junior ministers as »watchdogs « in portfolios that were captured by the smaller liberal coalition partner.

Marc Debus, Government formation, office allocation und coalition policy after the German federal election 2009: who could prevail in the Christian-liberal coalition government? 